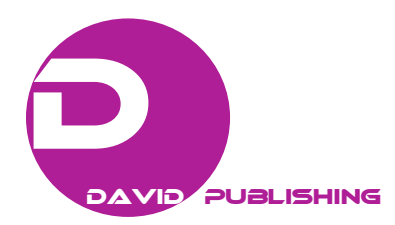

\title{
Visibility Enhancement during Severe Weather-Object Tracking with Bounding Box
}

\author{
Marwan S. Alluhaidan and Ikhlas Abdul-Qader \\ Electrical and Computer Engineering, Western Michigan University, Kalamazoo 49008, US
}

\begin{abstract}
A large number of vehicle accidents are caused by low visibility during severe weather conditions. According to the US Department of Transportation, there are more than 1,259,000 crashes each year caused by severe weather conditions. These accidents could be significantly prevented if real time visibility enhancement systems were to exist. This work is aimed to improve driver safety during severe weather conditions. We propose a method to improve driver's visibility during harsh weather conditions: a multistage framework based on Retinex algorithm and followed by a detection process of the vehicles in the front view of the driver. Retinex is an algorithm that has a capability to maintain a good dynamic range compression and spectral rendition effect. Retinex is used because it has the ability to overcome the loss of background details which occur during severe weather conditions scenes. We present simulation results of different visibility degradation. We also evaluate our method by using peak signal to noise ratio (PSNR) and Structural SIMilarity (SSIM) parameters.
\end{abstract}

Key words: Severe weather, visibility, Retinex, image enhancement.

\section{Introduction}

Low visibility is one of the major reasons for vehicles accidents in the United States. According to the US Department of Transportation there are more than 5,748,000 vehicle crashes each year. Approximately 1,259,000 crashes, almost $22 \%$ of the accidents, are caused by severe weather conditions such as heavy snow, rain, and fog. Over 445,303 people were injured and another 5,897 were killed in these accidents. Based on US National Highway Traffic Safety Administration (NHTSA) data, 17\% of the accidents occurred during snowy weather, over 38,700 vehicle crashes occur in fog, with over 600 people being killed and more than 16,300 people being injured in these crashes annually. Without a doubt, severe weather conditions significantly decrease road visibility and increase speed variance which leads to vehicle accidents [1].

The impact of bad weather such as snow and rain on visibility can make the difference between life and

Corresponding author: Marwan S. Alluhaidan, Ph.D., research fields: digital signal and image processing. death in a world that depends on satellite imagery and live feeds from surveillance cameras in policing and information gathering [2]. Severe weather conditions can significantly damage the image contrast and colors. Fig. 1 shows the effect of severe weather in image contrast. The effects of severe weather exponentially increase with the distance from the camera. As a result, consistently predictable space filtering techniques do not succeed in adequately removing varying and significant weather impacts. Lately, imaging under severe weather conditions has been under the spotlight and finally attracted interest in the vision and image processing communities. During bad weather conditions, the atmosphere severely scatters the light contacting the camera causing declining contrast variations throughout the scene. Indoor environments provide the ideal atmosphere for video capturing due to the formation of artificial illumination. In outdoor environments, the removal of weather effect is crucial in the creation of standard and quality images [3].

Severe weather impacts the quality and visibility of an image by interfering with the light waves in the 


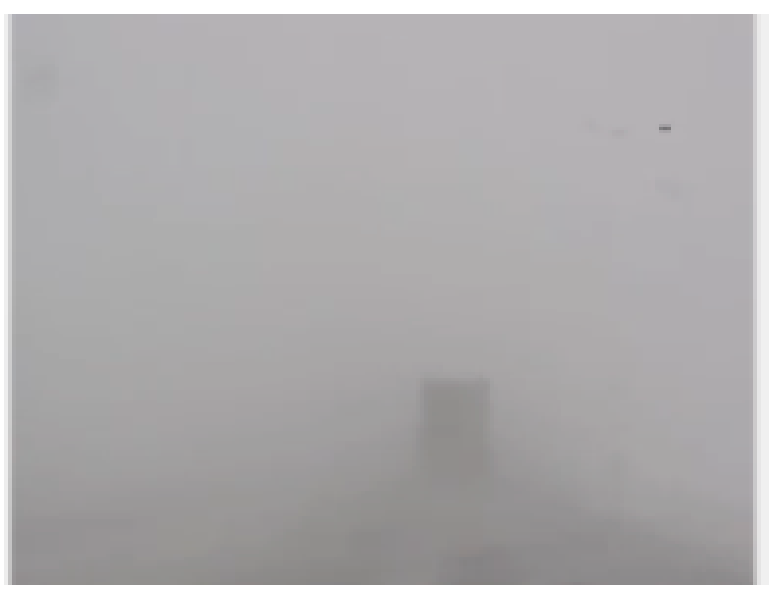

Fig. 1 Low visibility during snow storm.

atmosphere. The drops of rain and snowflakes deflect and reflect light waves away and towards the camera, which affects the illumination of the image. If raindrops and snowflakes come into direct contact with the camera, there is an increase in low visibility because of the contrasting perceptions in the image taken by the wet camera. Perception is affected because water and snow affects the intensity and color of an image and its surrounding regions differently. Altered perception can result in misjudgments on depth, distance, and time, which are important elements in gathering information from an image [4].

In our paper, we develop a novel framework to improve low visibility using storm scenes taken from dashboard videos. Our framework is based on Retinex and a validation of the proposed system is carried out by the implementation in MATLAB.

The key contributions of this paper are:

- A method that improves the low visibility caused by severe weather conditions to reduce the effect of low visibility on the driver's vision.

- Results of this method show a satisfactory performance to reduce low visibility and investigate vehicles in front of driver from images and video degraded by severe weather.

\section{Background}

Many researchers have proposed methods and algorithms to reduce the effects of severe weather by using image processing techniques. There are two main techniques of image enhancement that are normally applied: frequency and spatial domain. The frequency domain focuses on enhancing the frequency or the intensity in which grey values occur in an image. The frequency domain techniques work by increasing the magnitude of the high frequency components in an image in order to enhance clarity [5]. On the other hand, spatial domain focuses on the total number of pixels, which, make up an image. Unlike frequency domain techniques, spatial domain methods operate directly on individual pixels within the image. By focusing directly on the pixels, an observer can increase their understanding of the data in the image through manipulation. The image enhancement process in this case involves changing the grey scale pixels in the image to create a clearer image [6]. The following section briefly describes some of the techniques used to remove severe weather effects.

Frame difference was proposed by Huiying and Xuejing [7] and Garg and Nayar [8] as a method for detecting and removing rain and snow. They presented a method using vital physical structures of raindrops where the size generally varies between 0.1 and 3.5 $\mathrm{mm}$. They used three algorithms of frame difference method which is two-, three-, and five-frames and adding the constraints of area and direction angle of rain and snow [7]. The shortcoming of this method is that assuming the rain drop and snow is stationary and also the background should not be bright or white.

Rajderkar and Mohod [9] presented a rain and snow removal method using a single image and image decomposition. The image decomposition is mostly carried out through morphological component analysis using the morphological diversity of varying image data features. These features must pass through the process of putrefaction and then each constituent must be linked to articulate a lexicon of atoms. However, this method has a long procedure for the dictionary learning step. In addition, the output image is a gray scale image that has no color information [10]. 
$\mathrm{Xu}$ et al. [11] proposed a method that removing rain and snow in a single image by using guided filter. The fundamental idea for using the guided filter method involves the employment of transformation in foreground and background images to pinpoint active objects. If the subsequent variance after the deduction is greater than the guaranteed inception, then it defines a pixel to pixel movement target. The amount and bulk of model updates significantly affecting foreground effects. Significant changes in the object's background cannot be easily or rapidly overcome by slow adapting background models, for example a cloud passing above a scene. As a result, there exists a time frame where countless background pixels are classified inaccurately as foreground pixels. The process should be quick to avoid slow update rates that lean towards the creation of ghost masks that trail the real object. Quickly adjustable background models have the capacity to deal with background changes as they occur, but they crush at truncated frame rates. They are highly prone to noise as well as the aperture issue. The output image is blurred and also lost most of the image details and edges [11].

Zhen and Jihong [12] proposed a new approach to remove rain and snow from multiple degraded images using wavelet multi-level decomposition and wavelet fusion. The wavelet analysis is a severe weather removal method aimed at disintegrating signals into EEG sub-bands with varying tenacities, regularity, and directional features. The rain and snow elimination techniques of various images entail such aspects as the digital image and wavelet analysis as well as the determination of the rain/snow noise layer. Scenes of disintegrated images describe varying frequency from that of rain/snow noise, rain is greater than disintegrated image scenes. The consistency frequency and advantage of the division data is reasonably high and may even be greater than the one for rain, but that of background and pigment data of imageries is truncated. Another vital feature of the wavelet analysis is the fusion on multiple continuous degraded images, which identifies that rainy and snowy days represent dynamic and adverse weather that simplifies the process of the acquisition of multiple continuous varying disintegrated images. However, the results of this approach are not accurate enough [12].

Histogram techniques have been applied to remove severe weather from images and video by many researchers [13-17]. Histogram techniques work by reducing the frequency of the occurrence of different gray levels in an image. There most common techniques of Histogram are Histogram Equalization, Adaptive Histogram Equalization and Contrast Limited Adaptive Histogram Equalization (CLAHE) [13]. The aim of Histogram Equalization is to enhance the appearance of an image by stretching out the contrast of an image to attain an almost even distribution of gray values between the ranges of $0-255$ [14].

Retinex algorithm has been used in image enhancement to increase image contrast and intensities [4]. The Retinex Algorithm uses the principle of human sight, which is controlled by the retina and the cortex [2]. The human eye allows human beings to perceive light through a process of spatial comparison of the different rays of light allowed into the eyes [4]. Color perception of the human eye and correction by the retina and cortex ensures the capturing of a dynamic range of colors. Simply Retinex algorithms work by improving an image's dynamic range compression and tonal rendition [18]. This method has been developed through many different techniques which are Single Scale Retinex (SSR), Multi Scale Retinex (MSR) and Multi Scale Retinex with Color Restoration (MSRCR).

SSR works by either improving dynamic range compression or tonal rendition. The trade-off between dynamic range compression and tonal rendition is governed by the Gaussian distribution. The Gaussian distribution creates a space constant of 80 pixels between the dynamic range compression and tonal 
rendition [18]. The SSR algorithm is expressed as:

$$
\begin{gathered}
R_{i}(x, y)=\log I_{i}(x, y)-\log [F(x, y) \\
\left.* I_{i}(x, y)\right]
\end{gathered}
$$

where $I_{i}(x, y)$ is the distribution in the $i$ th color band of the image, $F(x, y)$ is the surround function. On the other hand, the Gaussian surround function is expressed as:

$$
(x, y)=K e^{-\left(x^{2}+y^{2}\right) / \sigma}
$$

where $\sigma^{2}$ is the variance and with $K$ selected so that:

$$
(x, y) d x d y=1
$$

The MSR is better than the SSR in image enhancement since it produces a better balance of dynamic compression and color rendition [19]. The MSR works like most image enhancement methods by lightening the image to increase contrast and dynamic range. Since the MSR output is simply the weighted sum of several SSR's with different scales, the MSR algorithm is expressed as:

$$
\begin{gathered}
R_{M S R i}=\sum_{n=1}^{N} \omega_{n}\left[\log I_{i}(x, y)-\log [F(x, y)\right. \\
\left.\left.* I_{i}(x, y)\right]\right]
\end{gathered}
$$

where $\omega_{n}$ is the weight for the $n$th scale and $N$ is number of scales.

The MSRCR technique restores intensity and color in an image and helps to improve perception of the image [2]. The MSRCR algorithm is expressed by:

$$
\begin{gathered}
R_{M S R C R i}(x, y)=C_{i}(x, y) R_{M S R i}(x, y) \\
C_{i}(x, y)=\beta \log \left[\alpha I_{i}^{\prime}(x, y)\right] \\
I_{i}^{\prime}(x, y)=I_{i}(x, y) / \sum_{i=1}^{S} I_{i}(x, y)
\end{gathered}
$$

where $\beta$ is gain constant, $\alpha$ controls the strength of the nonlinearity, and $s$ is the number of spectral channels.

Tsutsui et al. [20] proposed a method to reduce the halo artifacts and lower the computational costs associated with the Retinex algorithm. This method works by minimizing the cost function and is depicted as:

$$
\begin{aligned}
F[l]=\int_{\Omega}\left(|\nabla l|^{2}\right. & +\alpha(s-l)^{2} \\
& \left.+\beta|\nabla(s-l)|^{2}\right) d x d y
\end{aligned}
$$

where $\alpha$ and $\beta$ are parameters, $s(x, y)$ is input image, $r(x, y)$ is reflectance image, $l(x, y)$ is illumination image, $|\nabla l|^{2}$ is spatial smoothness of illumination image, $(s-l)^{2}$ is closeness between $l$ and $s$, and $|\nabla(s-l)|^{2}$ represents spatial smoothness of the reflectance image.

The Halo Artifacts Reduction method that is used for the variational-based Real-time Retinex Image Enhancement method is iterative in nature and works by finding the illumination image which minimizes the function $F[l]$ through an algorithm executed from the lowest resolution layer to the highest. This algorithm is referred to as the PNSD, which stands for projected normalized steepest descent [20].

In this method they used two approaches, which are edge adaptive parameters to estimate the illumination and erosion of the illumination. This method cannot enhance the dark regions near edges and the halo affect remains around those regions.

John and Wilscy [21] proposed an enhancement of video degraded by severe weather using wavelet fusion. Wavelet fusion enhances visibility, contrast while maintaining the color intensity. The image enhancement method comprises of three phases aimed at increasing the quality of the image while reducing the computational cost. In the first phase, the value of the atmospheric background radiation is calculated by augmenting the cost function of the algorithm. In the second phase, intensity data from the YIQ color model are used to estimate the airlight value. The first and second phases are critical in eliminating the whiteness from the image while adding depth. Once the first and second phases are complete, the wavelet fusion algorithm takes over to produce the final image which has more contrast, color fidelity, and dynamic compression. This method works well for application and does not require real time such as surveillance and object detection. 


\section{Methodology}

The proposed method aims to reduce low visibility from images or video in order to increase driver's visibility during low visibility conditions such as in storms. The method preserves most of the original image details. The proposed system is composed of 4 steps, which are preprocessing for initial conditioning, Retinex Enhancement Technique, postprocessing aiming for the detection, and tracking of cars in the front view of the vehicle. Fig. 2 is a flowchart of the proposed system.

\subsection{Preprocessing Stage}

Preprocessing operations will take place with the objective of not increasing the image information contrast, and in fact they will decrease it. In addition, these operations remove the noise or reduce it from the image/frame. Noise occurs in the image during the acquisition process, which affects the values and intensities of pixels. There are many different ways that noise can have an effect on an image/frame, depending on how the image/frame is originated [22]. There are many methods used to remove or reduce noise from an image/frame. In this step the Gaussian filter to reduce noise effect was used.

\subsection{Retinex Enhancement Technique}

In this step the Retinex technique is used to enhance and increase the visibility of images and frames. The Retinex theory decomposes the image into two images: illumination and reflectance. This decomposition allows us to control the illumination effects. The Retinex algorithm has been used in image enhancement for many years due to good results that have been achieved. The only shortcoming is the "halo" artifacts that appear on the edges. The Retinex algorithm works by applying surrounding function to estimate the background illumination. Then enhance the image by using logarithmic space operation. The surround function $F(x, y)$ has an essential effect on Retinex

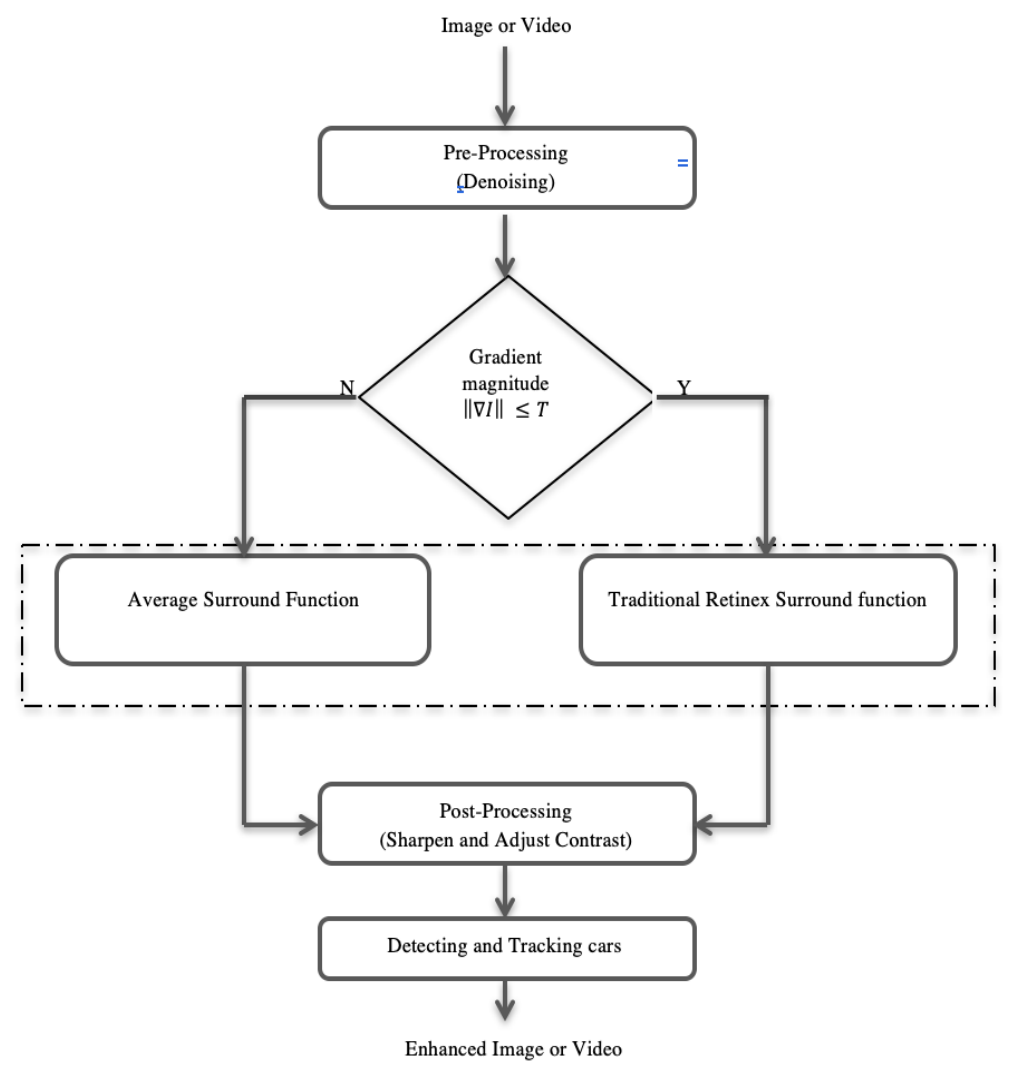

Fig. 2 Flowchart of proposed method. 
algorithm. The main purpose of a surround function is to estimate the illumination effects but it produces the "halo" artifacts on the significant edges [23]. The halo artifacts can be prevented by using averaging filter on the edges and keeping the same surround function on smooth area. The average expression can be expressed by:

$$
G(x, y)=\sum_{i} \sum_{j} I(x, y) W(x-i, y-j)
$$

The adaptive filter is designed according to the Roberts gradient operator. The Robert method finds edges using Robert approximation to the derivative. It returns edges at those points where the gradient of an image is maximum [24]. The gradient function is:

$$
\nabla \mathrm{I}(\mathrm{x}, \mathrm{y})=\left(\frac{\partial I}{\partial x}, \frac{\partial I}{\partial y}\right)
$$

where $\frac{\partial f}{\partial x}$ is the partial divertive for $x$ point and $\frac{\partial f}{\partial y}$ is the partial divertive for $y$ point. The edges are calculated by taking the magnitude of the gradient as shown in the formula:

$$
\|\nabla I\|=\sqrt{\left(\frac{\partial I}{\partial x}\right)^{2}+\left(\frac{\partial I}{\partial y}\right)^{2}}
$$

The adaptive filter makes it possible to enhance the visibility while keeping most of the image and frame details. In this method, the surround function $F_{A}(x, y)$ was used as follows:

$$
F_{A}(x, y)=\left\{\begin{array}{c}
F(x, y)=k e^{-\frac{x^{2}+y^{2}}{\sigma^{2}}}\|\nabla I\| \leq T \\
F(x, y)=\sum_{i} \sum_{j} W(x-i, y-j)\|\nabla I\|>T
\end{array}\right.
$$

\subsection{Postprocessing}

The post-processing step is the final step applied to enhance the quality of images or video. In this step, the contrast of images and frames were sharpened and adjusted. The Gaussian High Pass Filter (GHPF) is used for sharpener images and frames. This filter is used to enhance some details in images. The GHPF has the same effect as the ideal High Pass Filter (HPF) except that the GHPF has a smoother transition than the ideal HPF [25]. On the other hand, adjustments of the images and frames are used to improve the contrast and intensity values of the output.

\subsection{Detecting and Tracking Cars}

The last step is to detect and track the cars in front of the driver with bounding box. In this step, Gaussian Mixture Models (GMMs) and blob analysis were used to detect and track vehicles in Fig. 3. The Gaussian Mixture Model is a function of parametric probability density, which is represented as a weighted sum of Gaussian component densities. GMMs are used to separate the background and foreground pixels in images and frames. GMMs are commonly used for continuous measurements as a parametric model of the probability distribution. This algorithm works by comparing each pixel to the mean value. After comparing each pixel, the value of Gaussian weight, mean, and standard deviation values are updated to reflect the new obtained pixel value. Then threshold is applied to determine which pixels are foreground and background [26, 27].

\subsection{Blob Analysis}

A blob in image processing is defined as a group of connected pixels. This blob is used to trace the movement of an object within a frame. This algorithm can distinguish between foreground and background pixels by the value of each pixel. Also, it groups pixels that have similar values such as light values and color values to create the blob. The blob analysis works by searching through an entire image or frame and detecting all particles (blobs). Then build a detailed report of information for each blob including 


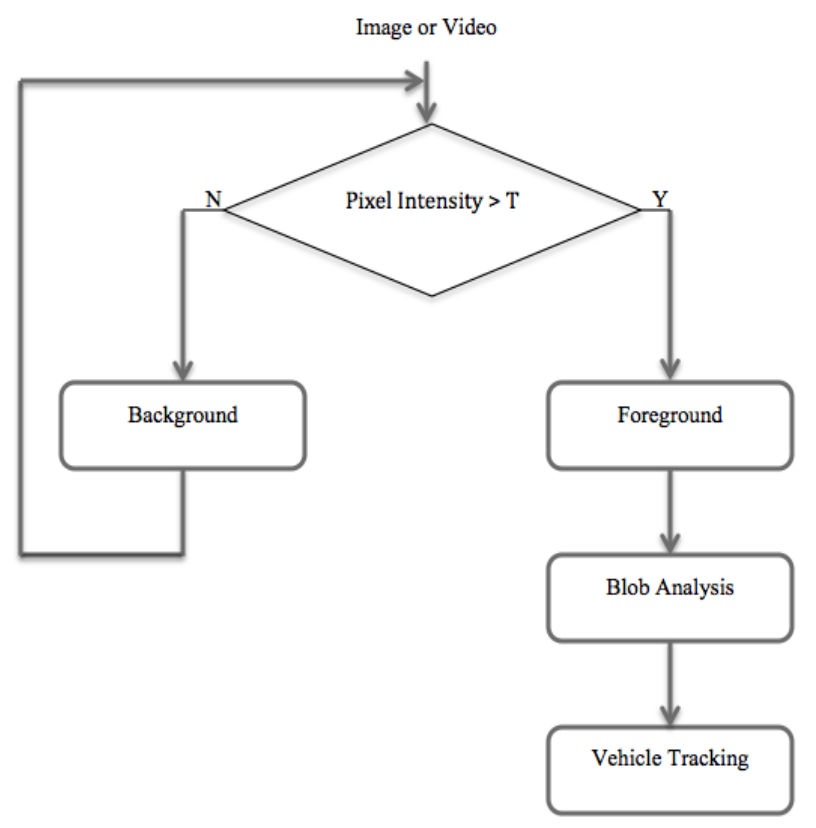

Fig. 3 Gaussian mixture model.

blob's location, size, and shape. After identifying the blobs contain vehicles in the image or frame, a rectangular box was put around them [27, 28].

\section{Simulation Results}

We validate our proposed method via MATLAB implementation. Frames extracted from videos taken from a dashboard camera during snowstorms were used. The video has a frame rate of $30 \mathrm{fps}$ and the frame size is equal to $512 \times 512 \times 3$. In addition, the frames have extreme low visibility as shown in Fig. 4. In this proposed method based on Retinex algorithm, it is assumed that the number of scales is equal to 3 , which is represented in the RGB colors. The weights were assumed to be equal to $1 / 3$ since true color is used. The average filter window size is chosen to be 3 $\times 3$. The proposed method was built using MATLAB software in computer with 4 GB memory RAM and 1.7 Intel® core $^{\mathrm{TM}}$ i7 CPU. The results show improved visibility in frames with extremely low visibility in Figs. 5-7.

\section{Discussion}

The proposed method's performance was compared with a histogram equalization and an MSR. Figs. 5-7

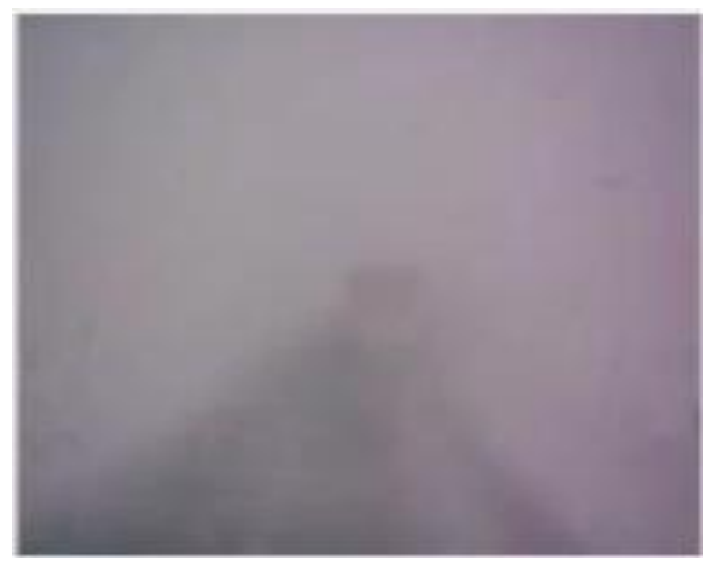

Fig. 4 Low visibility frame.

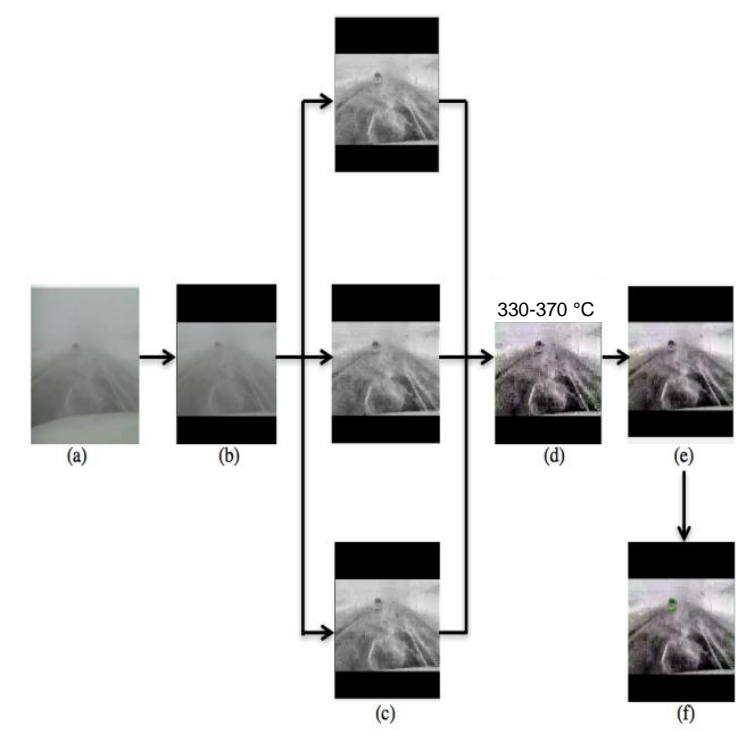

Fig. 5 Proposed method example: (a) Original Frame, (b) Gaussian LP Filter, (c) Red, Green and Blue Channel, (d) Combined Retinex Image and (e) Result Frame, (f) Result with bounding box around the vehicle.

show that frames have low visibility and are taken from videos during snowstorms. Figs. $5 \mathrm{~b}, 6 \mathrm{~b}$, and $7 \mathrm{~b}$ show frames after using a Gaussian low pass filter to reduce the noise that occurs during the acquisition process. Figs. $5 c, 6 c$ and $7 c$ demonstrate the three of channels red, green, and blue before running a Retinex algorithm. Frame results after running a Retinex algorithm are shown in Figs. 5d, 6d, and 7d. Figs. 5e, $6 e$, and $7 \mathrm{e}$ show the results after running a Gaussian high pass filter. The final results are shown in Figs. 5f, 6f, and $7 f$ after running the Gaussian mixture models and blob analysis to detect and track vehicles. 


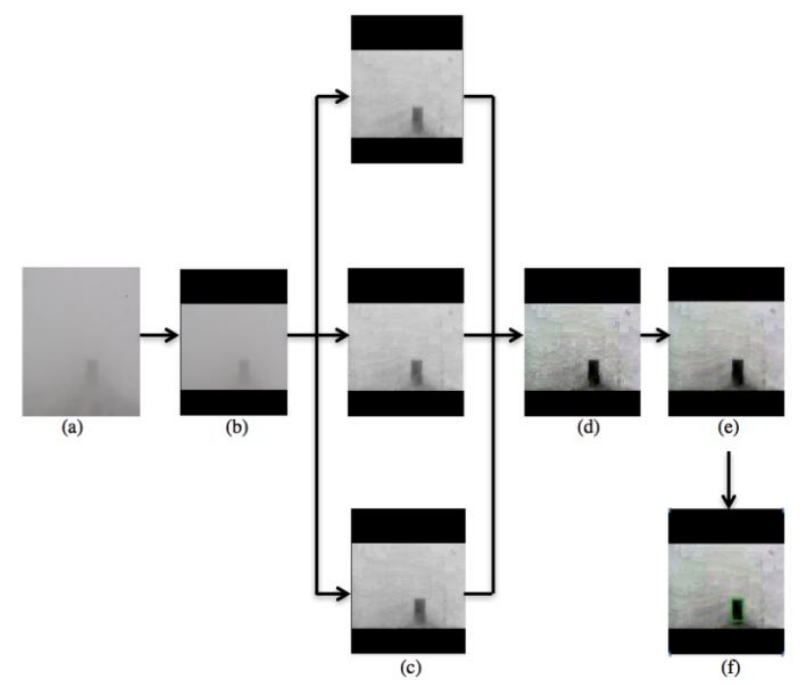

Fig. 6 Proposed method example: (a) Original Frame, (b) Gaussian LP Filter, (c) Red, Green and Blue Channel, (d) Combined Retinex Image and (e) Result Frame, (f) Result with bounding box around the vehicle.

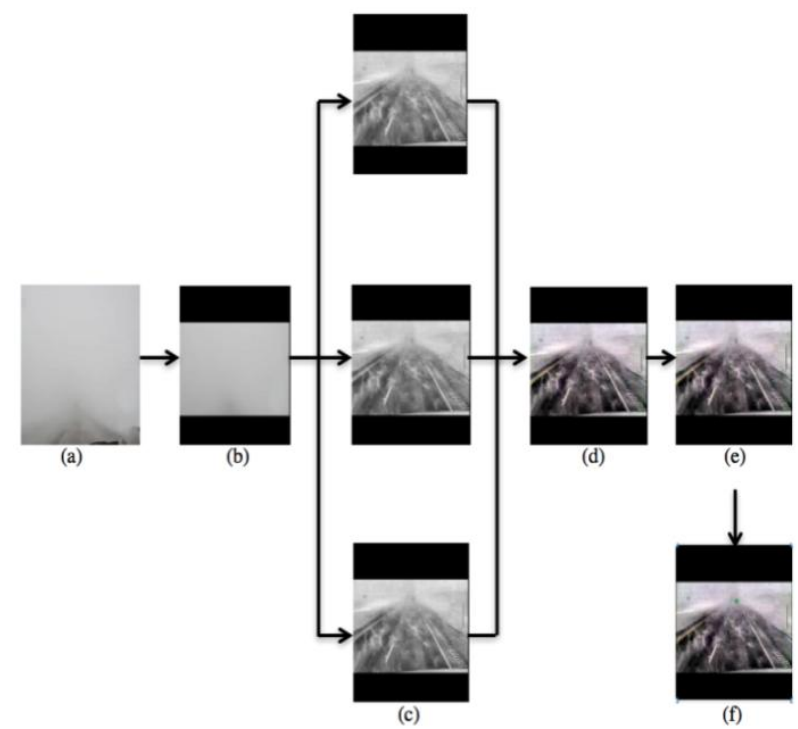

Fig. 7 Proposed method example: (a) Original Frame, (b) Gaussian LP Filter, (c) Red, Green and Blue Channel, (d) Combined Retinex Image and (e) Result Frame, (f) Result with bounding box around the vehicle.

To evaluate our proposed method, a peak signal to noise ratio (PSNR) and Structural SImilarity (SSIM) were used. The PSNR represents the ratio between the maximum power of a signal and the power of noise that affects the signal. PSNR is usually used for measuring the quality of reconstruction of lossy compression. SSIM is a method for measuring the quality of digital images and videos. SSIM is used for
Table 1 PSNR values for test images.

\begin{tabular}{llll}
\hline Figure & HE & MSR & Proposed method \\
\hline Fig. 5 & 57.95 & 55.19 & 65.69 \\
Fig. 6 & 57.99 & 55.25 & 67.38 \\
Fig. 7 & 56.49 & 55.04 & 64.94 \\
\hline
\end{tabular}

Table 2 SSIM calculation.

\begin{tabular}{llll}
\hline Figure & HE & MSR & Proposed method \\
\hline Fig. 5 & 0.52 & 0.74 & 0.83 \\
Fig. 6 & 0.55 & 0.75 & 0.89 \\
Fig. 7 & 0.50 & 0.72 & 0.81 \\
\hline
\end{tabular}

measuring between the result image and the initial image or reference image. Tables 1 and 2 show the PSNR and SSIM values for test images. The proposed method provides the PSNR and SSIM values greater than the histogram equalization and multi-scale Retinex methods.

\section{Conclusions}

Low visibility during severe weather limits the driver's vision and safety. Therefore, this work is concerned with finding a system to increase roads visibility during severe weather conditions. Using Retnix based framework, we provide a reliable system to reduce the effect of severe weather on visibility. This system increases visibility during severe weather and can be used in a wide range of applications including drivers' safety.

There are several additional areas for further research to improve this method. These include finding more powerful and general procedure for noise removing to reduce the need of pre- or post-processing step. Also, further parallelization of the code could decrease the execution time of this method.

\section{References}

[1] "How Do Weather Events Impact Roads? FHWA Road Weather Management." (n.d.). Retrieved from https://ops.fhwa.dot.gov/weather/q1_roadimpact.htm.

[2] Livingston, M. A., Garrett, C. R., and Ai, Z. 2011. Image Processing for Human Understanding in Low-Visibility. Naval Research Lab Washington DC Information Technology Div.

[3] Alluhaidan, M. S., and Abdel-Qader, I. 2016. 
"Retinex-Based Visibility Enhancement System during Inclement Weather." In Computational Science and Computational Intelligence (CSCI), 2016 International Conference, pp. 688-91.

[4] Alluhaidan, M. S., \& Abdel-Qader, I. (2018). Visibility Enhancement in Poor Weather-Tracking of Vehicles. In Proceedings of the International Conference on Scientific Computing (CSC) (pp. 183-188). The Steering Committee of the World Congress in Computer Science, Computer Engineering and Applied Computing (WorldComp).

[5] Simon, N., and Kumar, A. 2014. "Contrast Enhancement of Color Images Using Improved Retinex Method." International Journal of Research in Engineering and Technology 3 (11): 279-85.

[6] Ramkumar, M., and Karthikeyan, B. 2013. "A Survey on Image Enhancement Methods.” International Journal of Engineering and Technology (IJET) 5 (2): 960-1012.

[7] Khehra, M. K., and Devgun, M. 2015. "Survey on Image Enhancement Techniques for DigitalImages." Scholars Journal of Engineering and Technology 3 (2): 202-6.

[8] Huiying, D., and Xuejing, Z. 2015. "Detection and Removal of Rain and Snow from Videos Based on Frame Difference Method." In Control and Decision Conference (CCDC), 2015 27th Chinese, pp. 5139-43.

[9] Garg, K., and Nayar, S. K. 2004. "Detection and Removal of Rain from Videos." In Computer Vision and Pattern Recognition, 2004. CVPR 2004, Proceedings of the 2004 IEEE Computer Society Conference, Vol. 1, p. 1.

[10] Rajderkar, D., and Mohod, P. S. 2013. "Removing Snow from an Image via Image Decomposition.” In Emerging Trends in Computing, Communication and Nanotechnology (ICE-CCN), $2013 \quad$ International Conference, pp. 576-9.

[11] Fu, Y. H., Kang, L. W., Lin, C. W., and Hsu, C. T. 2011. "Single-Frame-Based Rain Removal via Image Decomposition." In Acoustics, Speech and Signal Processing (ICASSP), 2011 IEEE International Conference, pp. 1453-6.

[12] Xu, J., Zhao, W., Liu, P., and Tang, X. 2012. "Removing Rain and Snow in a Single Image Using Guided Filter." Computer Science and Automation Engineering 2: 304-7.

[13] Zhen, C., and Jihong, S. 2013. "A New Algorithm of Rain (Snow) Removal in Video." Journal of Multimedia 8 (2): 168.

[14] Kaur, M., Kaur, J., and Kaur, J. 2011. "Survey of Contrast Enhancement Techniques Based on Histogram Equalization." International Journal of Advanced Computer Science and Applications 2 (7): 137-41.

[15] Chaudhary, C., and Patil, M. K. 2013. "Review of Image Enhancement Techniques Using Histogram Equalization." International Journal of Application or Innovation in
Engineering and Management 2 (5): 1297-301.

[16] Vij, K., and Singh, Y. 2009. "Enhancement of Images Using Histogram Processing Techniques." Int. J. Comp. Tech. Appl. 2 (2): 309-13.

[17] Kapoor, K., and Arora, S. 2015. "Colour Image Enhancement Based on Histogram Equalization." Electrical \& Computer Engineering: An International Journal 4 (3): 73-82.

[18] Bubakar, F. M. 2012. "Image Enhancement Using Histogram Equalization and Spatial Filtering."

[19] Rahman, Z. U., Jobson, D. J., and Woodell, G. A. 1996. "Multi-scale Retinex for Color Image Enhancement." In Image Processing, 1996. Proceedings, Vol. 3, pp. 1003-6.

[20] Rahman, Z. U., Jobson, D. J., and Woodell, G. A. 2004. "Retinex Processing for Automatic Image Enhancement." Journal of Electronic Imaging 13 (1): 100-10.

[21] Tsutsui, H., Yoshikawa, S., Okuhata, H., and Onoye, T. 2012. "Halo Artifacts Reduction Method for Variational Based Realtime Retinex Image Enhancement.” In Signal \& Information Processing Association Annual Summit and Conference (APSIPA ASC), 2012 Asia-Pacific, pp. 1-6.

[22] John, J., and Wilscy, M. 2008. "Enhancement of Weather Degraded Video Sequences Using Wavelet Fusion.” In Cybernetic Intelligent Systems, 2008. CIS 2008. 7th IEEE International Conference, pp. 1-6.

[23] Rao, Y., and Chen, L. 2012. "A Survey of Video Enhancement Techniques." Journal of Information Hiding and Multimedia Signal Processing 3 (1): 71-99.

[24] Hu, X., Gao, X., and Wang, H. 2014. "A Novel Retinex Algorithm and Its Application to Fog-Degraded Image Enhancement." Sensors \& Transducers 175 (7): 138.

[25] Jain, R., Kasturi, R., and Schunck, B. G. 1995. Machine Vision, Vol. 5. New York: McGraw-Hill.

[26] MatWorks, T. 1994. "Contrast Adjustment." http://www.mathworks.com/help/images/contrast-adjust ment.html. Accesses: Sep. 8, 2016.

[27] Santosh, D. H. H., Venkatesh, P., Poornesh, P., Rao, L. N., and Kumar, N. A. 2013. "Tracking Multiple Moving Objects Using Gaussian Mixture Model.” International Journal of Soft Computing and Engineering (IJSCE) 3 (2): 114-9.

[28] Chiranjeevi, A., and Munappa, S. "A Hybrid Algorithm for Vehicle Detection and Tracking." International Journal of Engineering Technology, Management and Applied Sciences 3 (9).

[29] Hari Hara Santosh, D., Venkatesh, P., Poornesh, P., Narayana Rao, L., and Arun Kumar, N. 2013. "Tracking Multiple Moving Objects Using Gaussian Mixture Model." International Journal of Soft Computing and Engineering 3 (2): 114-8. 